\title{
Parameterization of written signatures based on EFD
}

\author{
Pere Marti-Puig, Jaume Danés, Jordi Solé-Casals \\ ${ }^{I}$ Group of Digital Technologies, University of Vic, C/ de Laura 13, 08500 Vic (Barcelona),Spain \\ pere.marti@uvic.cat, jaume.danes@uvic.cat, jordi.sole@uvic.cat
}

\begin{abstract}
Keywords: Quantitative shape analysis, Elliptical Fourier Descriptors (EFD), handwriting recognition, biometrics.
Abstract: $\quad$ In this work we propose a method to quantify written signatures from digitalized images based on the use of Elliptical Fourier Descriptors (EFD). As usually signatures are not represented as a closed contour, and being that a necessary condition in order to apply EFD, we have developed a method that represents the signatures by means of a set of closed contours. One of the advantages of this method is that it can reconstruct the original shape from all the coefficients, or an approximated shape from a reduced set of them finding the appropriate number of EFD coefficients required for preserving the important information in each application. EFD provides accurate frequency information, thus the use of EFD opens many possibilities. The method can be extended to represent other kind of shapes.
\end{abstract}

\section{INTRODUCTION}

The quantitative shape analysis that is sometimes required in biometrics, agronomy, medicine, genetics, ecology or taxonomy, among other research fields, is commonly performed on the contours extracted from images (Lestrel, 1997). One of the major problems when performing an automatically quantification of contour sets is the large amount of data involved in describing the shape. As a result, previous to the application of a known analysis or classification technique, the contours are parameterized. Then, with suitable contour parameterization, the most relevant shape information for a particular purpose can be represented with a reduced number of coefficients. Although different contour descriptors have been developed, the most widely used are the Elliptical Fourier Descriptors (EFD) that are applied to the $(x, y)$ contour coordinates. EFD were first proposed by Kuhl and Giardina (Kuhl and Giardina, 1982) and one of the reasons for its wide acceptance is because EFD can represent all kinds of close curves as well as preserve the original shape information when shape reconstruction is required, using only a limited number of coefficients, providing intuitive information about the number of coefficients required to preserve a given level of detail of the shapes. EFD can also be prepared to be invariant to translation, rotation and scale (Nixon and Aguado,
2008). There exist many fields that use EFDs for shape quantization. We found some examples applied to the characterization of biological contours of animals (Rohlf and Archie, 1984; Bierbaum and Ferson, 1986; Diaz et al. 1989; Ferson et al., 1985; Castonguay et al., 1991; Chen et al., 2000; Tort, 2003; Tracey et al. 2006) and applied to the contours of plants (Iwata et al., 2000; Iwata et Ukai, 2002; Iwata et al., 2004). Concerning the practical uses of EFD, although the reconstruction of any discrete contour can be perfect with the appropriate number of EFD coefficients, in realistic applications a good balance between the preservation of the relevant shape information and interesting data dimensional reduction must be done. Hence, only a part of the coefficients are selected.

\section{ELLIPTICAL FOURIER CONTOUR DESCRIPTORS OVERVIEW}

As it is well-known, a continuous close contour with period $\mathrm{T}$ is defined by the evolution of its coordinates $x(t)$ and $y(t)$ along the variation of $t$. The contour coordinates can be expanded using the Fourier series. The contour coordinates, in its equivalent real or complex forms, can be written as: 


$$
\begin{aligned}
x(t) & =a_{0}+\sum_{k=1}^{\infty}\left[a_{k} \cos \left(\frac{2 \pi k t}{T}\right)+b_{k} \sin \left(\frac{2 \pi k t}{T}\right)\right] \\
& =\sum_{k=-\infty}^{k=\infty} u_{k} e^{j \frac{2 \pi k k}{T} t} \\
y(t) & =c_{0}+\sum_{k=1}^{\infty}\left[c_{k} \cos \left(\frac{2 \pi k t}{T}\right)+d_{k} \sin \left(\frac{2 \pi k t}{T}\right)\right] \\
& =\sum_{k=-\infty}^{k=\infty} v_{k} e^{j \frac{2 \pi k k}{T} t}
\end{aligned}
$$

where:

$$
\begin{gathered}
u_{k}=\frac{1}{T} \int_{T} x(t) e^{-j \frac{2 \pi k}{T} t} d t \\
v_{k}=\frac{1}{T} \int_{T} y(t) e^{-j \frac{2 \pi k}{T} t} d t \\
\text { with: } \\
a_{k}=u_{k}+u_{k}^{*} \text { and } b_{k}=j\left(u_{k}-u_{k}^{*}\right) \\
c_{k}=v_{k}+v_{k}^{*} \text { and } d_{k}=j\left(v_{k}-v_{k}^{*}\right)
\end{gathered}
$$

The real coefficients $a_{k}, b_{k}, c_{k}$ and $d_{k}$ become an alternative to perfectly describe the contour and are known as Elliptic Fourier coefficients. It is easy to see from (3) and (4) that the coefficients $a_{0}$ and $c_{0}$ only represent the position of the centre of gravity of the shape contour. If $a_{0}$ and $c_{0}$ take the zero value the contour is centred in the origin. The contour approximation based on the EFD is achieved by selecting a reduced set of coefficients, i.e. by limiting the number of harmonics in the following way:

$$
\begin{aligned}
& x_{K}(t)=a_{0}+\sum_{k=1}^{K}\left[a_{k} \cos \left(\frac{2 \pi k t}{T}\right)+b_{n} \sin \left(\frac{2 \pi k t}{T}\right)\right] \\
& y_{K}(t)=c_{0}+\sum_{k=1}^{K}\left[c_{k} \cos \left(\frac{2 \pi k t}{T}\right)+d_{k} \sin \left(\frac{2 \pi k t}{T}\right)\right]
\end{aligned}
$$

Clearly the approximations $x_{K}(t)$ and $y_{K}(t)$ improve as $K$ increases.

\section{EFC DESCRIPTORS AND ITS RELATION TO THE DFT}

The contours from 2D images have a discrete nature and what we really have are the discrete signals $x(n)$ and $y(n)$ which can be thought of as sampled versions of $x(t)$ and $y(t)$ at the instants $t=n T / N$ where $\mathrm{n}$ goes from 0 to $N-1$. In practice, then, we have the $\mathrm{N}$ pair of points $(x(n), y(n))$ of a fundamental period. In order to show how the Fourier series expansion of the discrete signals $x(n)$ and $y(n)$ can be related to the DFT let us first consider the expansion of $x(t)$ in (1) and its complex equivalent expression in the following way:

$$
\begin{aligned}
x(t) & =a_{0}+\sum_{k=1}^{\infty}\left[a_{k} \cos \left(\frac{2 \pi k t}{T}\right)+b_{k} \sin \left(\frac{2 \pi k t}{T}\right)\right] \\
& =\sum_{k=-\infty}^{k=\infty} u_{k} e^{j \frac{2 \pi k}{T} t}=u_{o}+\sum_{k=1}^{k=\infty}\left(u_{k} e^{j \frac{2 \pi k}{T} t}+u_{-k} e^{-j \frac{2 \pi k}{T} t}\right)
\end{aligned}
$$

With the aim of obtaining the discrete signal $x(n)$ and $y(n)$ from the continuous ones, the following changes must be applied. First t has to be replaced by the discrete values $n T / N(n=0, \ldots, N-1)$ in (9). Second, taking into account that the discrete lowest frequency able to represent is $\omega_{1}=2 \pi / N$ and the highest is $\omega_{k}=\pi$ the set of analogical frequencies $\omega_{k}$ $=2 \pi k / T$ from $k$ going from 0 to $\infty$ becomes $\omega_{k}$ $=2 \pi k / N$ where index $k$ goes from 0 to $N / 2$. Then $x(n)$ takes the form:

$$
\begin{aligned}
x(n) & =a_{0}+\sum_{k=1}^{N / 2}\left[a_{k} \cos \left(\frac{2 \pi}{N} k n\right)+b_{k} \sin \left(\frac{2 \pi}{N} k n\right)\right] \\
& =\sum_{k=-N / 2}^{k=N / 2} u_{k} e^{j \frac{j \pi}{N} k n}=u_{o}+\sum_{k=1}^{k=N / 2}\left(u_{k} e^{j \frac{2 \pi}{N} k n}+u_{-k} e^{-j \frac{2 \pi}{N} k n}\right)
\end{aligned}
$$

In the same way, the coefficients $u_{k}$ can be found from (3) by replacing the integral by a summation, the continuous variable $\mathrm{t}$ by its samples $n T / N$ and $d t$ by the minimum increment $T / N$ :

$$
u_{k}=\frac{1}{T} \sum_{n=0}^{N-1} x(n) e^{-j \frac{2 \pi}{T} k\left(\frac{T n}{N}\right)}\left(\frac{T}{N}\right)=\frac{1}{N} \sum_{n=0}^{N-1} x(n) e^{-j \frac{2 \pi}{N} k n}
$$

Then, from the DFT definition, the coefficients $u_{k}$ can be obtained as:

$$
u_{k}=\frac{1}{N} X(k)
$$

Where $X(k)$ is the $k$-th element of the DFT of $x(n)$.

Let us remember the well-known conjugate symmetry DFT property (Proakis and Manolakis 1996):

$$
X(-k)=X^{*}(k)=X(N-k)
$$


This means that $u_{-k}=u_{k}{ }^{*}$ (where symbol * denotes the conjugation operation), then using (13) the last term of (11) becomes:

$$
x(n)=u_{o}+\sum_{k=1}^{k=N / 2}\left(u_{k} e^{j \frac{2 \pi}{N} k n}+u_{k}^{*} e^{-j \frac{2 \pi}{N} k n}\right)
$$

By writing $u_{k}$ in the polar form $u_{k}=\left|u_{k}\right| \mathrm{e}^{\mathrm{j} \phi \mathrm{k}}$, using the Euler formula and applying the well known equality $\cos (A+B)=\cos (\mathrm{A}) \cos (\mathrm{B})-\sin (\mathrm{A}) \sin (\mathrm{B})$, we have:

$$
\begin{aligned}
x(n) & =u_{o}+\sum_{k=1}^{k=N / 2}\left|u_{k}\right|\left(e^{j \frac{2 \pi}{N} k n+j \varphi_{k}}+e^{-j \frac{2 \pi}{N} k n-j \varphi_{k}}\right) \\
& =u_{o}+\sum_{k=1}^{k=N / 2} 2\left|u_{k}\right| \cos \left(\frac{2 \pi}{N} k n+\varphi_{k}\right) \\
& =u_{o}+\sum_{k=1}^{k=N / 2} 2\left|u_{k}\right| \cos \left(\varphi_{k}\right) \cos \left(\frac{2 \pi}{N} k n\right)-2\left|u_{k}\right| \sin \left(\varphi_{k}\right) \sin \left(\frac{2 \pi}{N} k n\right)
\end{aligned}
$$

As a result, from (11) and (15) it is easy to obtain the EFD coefficients of $x(n)$ from the DFT of $x(n)$. Similarly, as $y(n)$ has an expression formally identical to $x(n)$ the coefficients $c_{k}$ and $d_{k}$ are also obtained from the DFT of $y(n)$. Therefore we have:

$$
\begin{gathered}
a_{0}=u_{0}=\frac{X(0)}{N} \\
c_{0}=v_{0}=\frac{Y(0)}{N} \\
a_{k}=2\left|u_{k}\right| \cos \left(\varphi_{k}\right)=\frac{2 \operatorname{Re}[X(k)]}{N} \\
b_{k}=-2\left|u_{k}\right| \sin \left(\varphi_{k}\right)=-\frac{2 \operatorname{Im}[X(k)]}{N} \\
c_{k}=2\left|v_{k}\right| \cos \left(\varphi_{k}\right)=\frac{2 \operatorname{Re}[Y(k)]}{N} \\
d_{k}=-2\left|v_{k}\right| \sin \left(\varphi_{k}\right)=-\frac{2 \operatorname{Im}[Y(k)]}{N}
\end{gathered}
$$

where $X(k)$ and $Y(k)$ are the DFTs of $x(n)$ and $y(n)$ respectively. The discrete signal approximation of $x_{K}(n)$ and $y_{K}(n)(K<N / 2)$ takes the form as:

$$
\begin{aligned}
& x_{K}(n)=a_{0}+\sum_{k=1}^{K}\left[a_{k} \cos \left(\frac{2 \pi}{N} k n\right)+b_{k} \sin \left(\frac{2 \pi}{N} k n\right)\right] \\
& y_{K}(n)=c_{0}+\sum_{k=1}^{K}\left[c_{k} \cos \left(\frac{2 \pi}{N} k n\right)+d_{k} \sin \left(\frac{2 \pi}{N} k n\right)\right]
\end{aligned}
$$

Note that the DFT can be efficiently computed by its fast algorithm, the FFT, which can also be optimised for real data (Proakis and Manolakis
1996). Note also that the elliptic Fourier descriptors can be modified to be invariant with respect to size, rotation, or starting point (Nixon and Aguado 2008).

On the left of figure 1, a $2 \mathrm{D}$ butterfly contour is represented and on the right the sequences of 1024 points of $x(n)$ and $y(n)$ are depicted. In figure 2, following the same distribution, we can see the contour reconstruction, on the left, and the $x(n)$ and $y(n)$ reconstructions taken only the first 72 coefficients, 36 for $x(n)$ and $36 y(n)$, on the right.
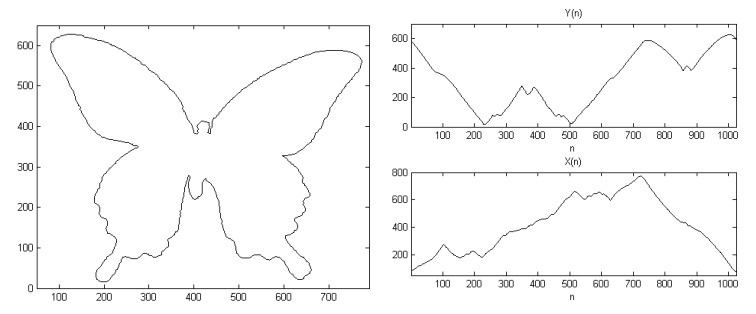

Figure 1: On the left, image contour. On the righ, representation of the contour coordinates $y(n)$ and $x(n)$.

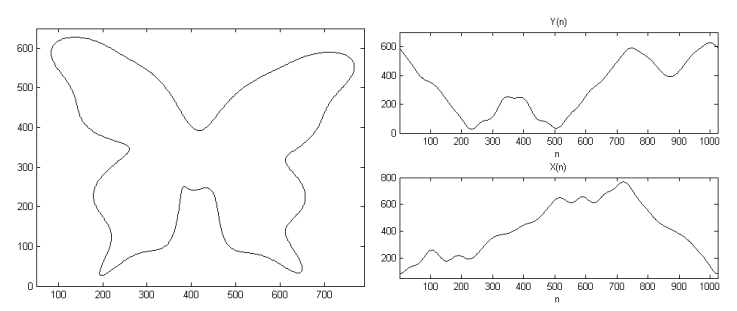

Figure 2: On the left, image contour reconstruction using EFD given 72 real coefficients. On the right, representation of the contour coordinates reconstruction employing the first 36 real coefficients for both $x(n)$ and $y(n)$.

In some applications it is interesting to obtain the coefficients representing a contour independently of position, scale factors or rotations. From (16) and (17), according to Nixon and Aguado (Nixon and Aguado, 2008), it is easy to see than the coefficients $a_{0}$ and $b_{0}$ represent the centre of the contour. By imposing $a_{0}=b_{0}=0$ the contour reconstruction is centred at the origin of coordinates. So, this is the condition to modify the parameters to be independent of the position. To be scale invariants, normalization of the set of parameters by $S$ is required, being $S=\max \left\{a_{l}, b_{1}, c_{l}, d_{l}\right\}$ (Nixon and Aguado 2008). The invariance to rotation requires defining a new set of parameters in the following way (Nixon and Aguado 2008): 


$$
r_{K}=\frac{a_{k}^{2}+b_{k}^{2}}{c_{k}^{2}+d_{k}^{2}}=\frac{\left|u_{k}\right|^{2}}{\left|v_{k}\right|^{2}}
$$

\section{PROPOSED PARAMETERIZATION METHOD}

In order to compare and quantify written signatures we will use images. A digital camera or a scanner can be used to obtain the images of the signatures. If the image is taken with a minimum quality by simple image processing techniques we obtain the different closed contours of the signature. Each signature can contain a particular number of closed contours with different number of points. The basic idea of the proposed method is the following: a signature is decomposed in different closed contours that are ordered from the longest to the lower. Then we consider only the $M$ longer contours. Each of these contours is parameterized by a reduced number of $N$ EFD coefficients that are ordered sequentially. When the signature has only $L$ closed contours, $L<M$, the last $(M-L) N$ parameters will be zero. In figure 3 the level of the EFD coefficients with respect to its index $(k)$ is presented, showing that the coefficients with lower index are more important than the coefficients with higher index. If we consider the characterization of the $\mathrm{N}$ most important contours per signature, and a signature has less than $\mathrm{N}$ contours we complete the difference with zeros. Depending on the application, we can consider the modification of the parameters in order to achieve invariance to scale and rotation, maintaining a relative position of the closed contours with respect to the centre of masses of the signature. We show the process by an example.

In figure 4 we show the reconstruction of a signature using 40, 30, 20 and 10 complex coefficients per contour.

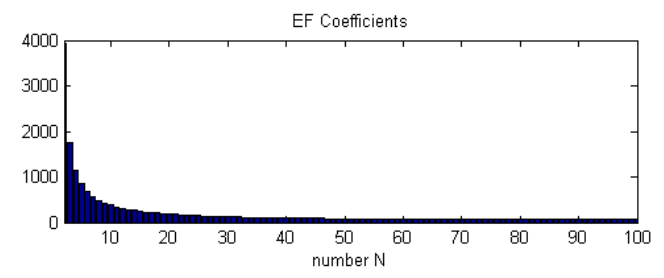

Figure 3: Representation of the EFD coefficients with its index.
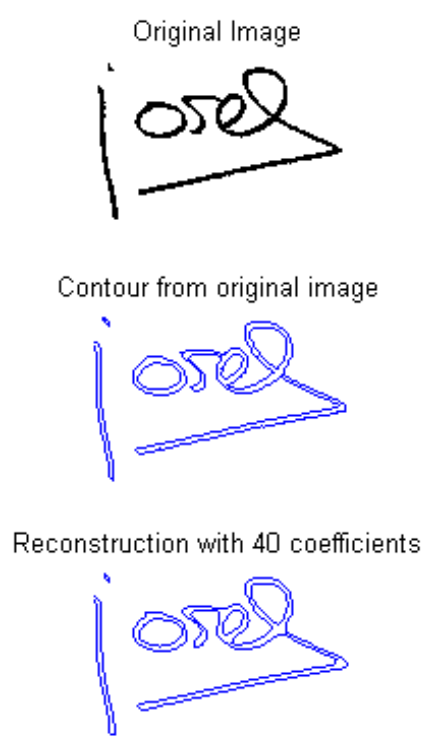

Reconstruction with 30 coefficients

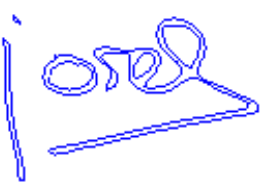

Reconstruction with 20 coefficients

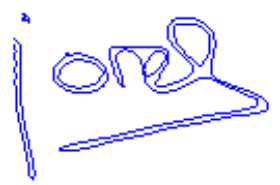

Reconstruction with 10 coefficients

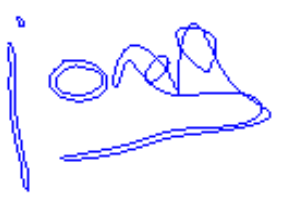

Figure 4: Representation of the original image, the contour extracted from the original image and some reconstructions using different number of EFD coefficients.

The signature used in figure 5 has only 7 contours. On the following figures (6 and 7), for the same example, we show the detail of 3 of these contours with the axis indicating position information. 


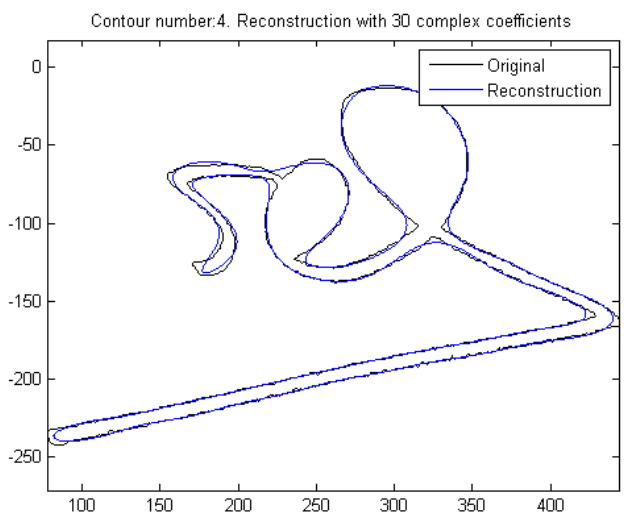

Figure 5: The longer contour with its reconstruction using 30 complex coefficients.

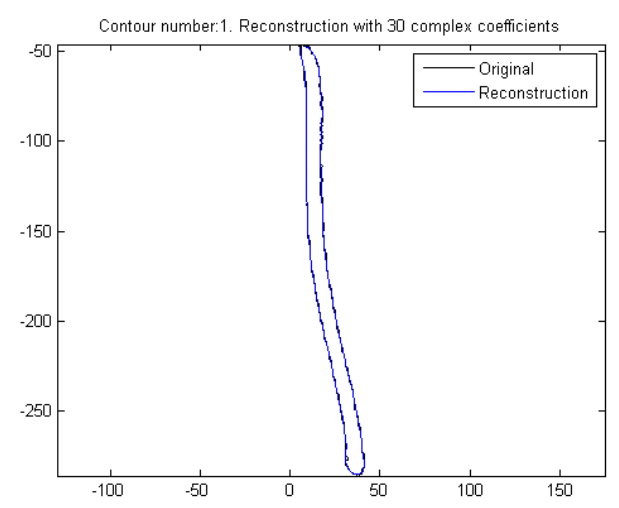

Figure 6: The second longer contour with its reconstruction using 30 complex coefficients.

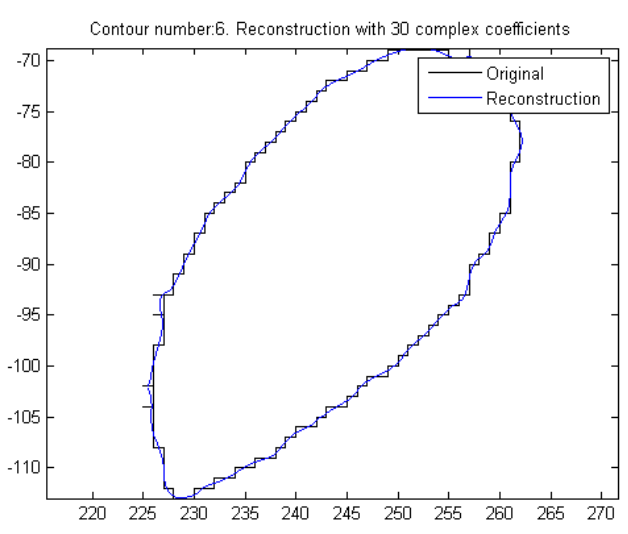

Figure 7: Another contour and its reconstruction.

Figures 8 provide some other kind of signatures with its reconstructions.
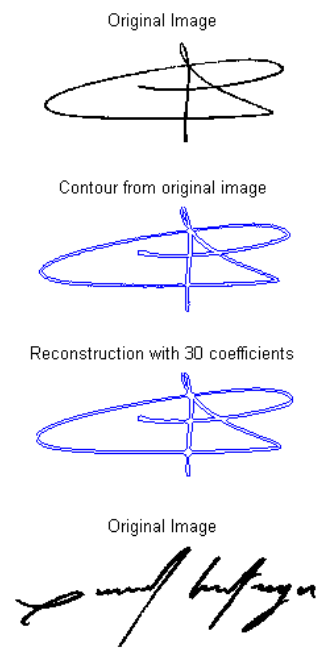

Contour from original image

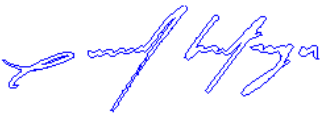

Reconstruction with 30 coefficients

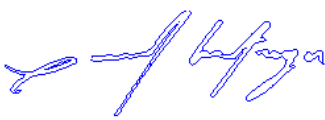

Original Image

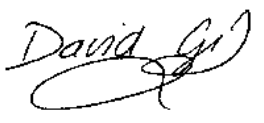

Contour from original image

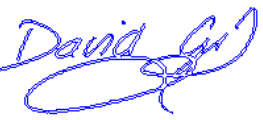

Reconstruction with 30 coefficients

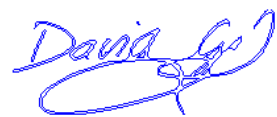

Original Image

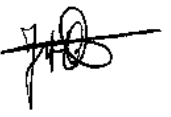

Contour from original image

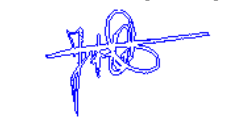

Reconstruction with 30 coefficients

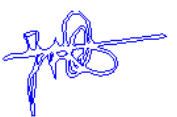

Figure 8: Another contour ad its reconstruction. 


\section{DISCUSSION AND FUTURE WORK}

The proposed parameterization can be easily used for characterizing signatures or words of different people. As the original images are static images (scanned of photo images), the kind of applications that can profit of this technique are related to off-line handwritten signature verification. One of the challenging problems in this field is the feature extraction process. Our system can be a good strategy in order to improve verification results and specially in order to develop a general system to classify every signature. Therefore, we are now putting our efforts in exploring the generalization capability of our extracted parameters for signature verification and writer recognition using EFD as parameters and different kind of linear and nonlinear classifier. It is important to note that writer recognition using short words has been recently studied in (Sesa-Nogueras and Faundez-Zanuy, 2012) and very good results have been obtained for text-dependent writer recognition.

\section{CONCLUSIONS}

In this work we show a way to apply the Elliptic Fourier analysis to the parameterization of signatures. We analyse also the relation of the coefficients given by Kuhl and Giardiana with the complex coefficients computed with the FFT in order to obtain a fast method for the EFD derivation. An important advantage when using the proposed kind of parameters is that from a reduced set of coefficients we can obtain an approximate reconstruction of the original shape, controlling the preserved or discarded information in order to optimize the performance for each application. One of the advantages of this method is that it allows comparison of different signatures of the same person taken in different times.

\section{ACKNOWLEDGEMENTS}

This work has been supported by the University of Vic under the grant R0904.

\section{REFERENCES}

Bierbaum R.M., Ferson S, 1986. Do symbiotic pea crabs decrease growth rate in mussels? Biol Bull. 170:51-61.

Castonguay, M., Simard, P., and Gagnon, P. (1991) Usefulness of Fourier analysis of otolith shape for Atlantic mackerel (Scomber scombrus) stock discrimination. Can.J.Fish.Aquat.Sci. 48(2):296-302.

Chen, S.Y.Y., Lestrel, P.E., Kerr, W.J.S., McColl, J.H., (2000) Describing shape changes in the human mandible using elliptical Fourier functions. Eur. J. Orthodont. 22, 205-216

Diaz G, Zuccarelli A, Pelligra I, Ghiani A, 1989. Elliptic Fourier analysis of cell and nuclear shapes. Comp Biomed Res. 22:405-414.

Ferson S, Rohlf FJ, Koehn RK, 1985. Measuring shape variation of two-dimensional outlines. Syst Zool. 34:59-68.

Iwata $\mathrm{H}$, Niikura $\mathrm{S}$, Matsuura S, Takano Y, Ukai Y, (2000). Diallel analysis of root shape of Japanese radish (Raphanus sativus L.) based on elliptic Fourier descriptors. Breed Sci. 50:73-80.

Iwata, H., Ukai, Y., (2002) SHAPE: a computer program package for quantitative evaluation of biological shapes based on elliptic Fourier descriptors. J. Hered. 93, 384-385.

Iwata, H., S. Niikura, S. Matsuura, Y. takano and Y. Ukai (2004) Interaction between genetic effects and soil type in diallel analysis of root shape and size of Japanese radish (Raphanus sativus L.). Breeding Science 54: 313-318.

Kuhl, F.P., Giardina, C.R., (1982) Elliptic Fourier features of a closed contour. Comput. Graph. Image Process. 18, 236-258.

Lestrel, P.E. (Ed.)., (1997) Fourier Descriptors and their Application in Biology. Cambridge University Press, UK, p. 466.

Nixon, M., Aguado A. (2008) Feature Extraction and Image Processing (2 Ed.) Academic Press.

Rohlf FJ, Archie JW, (1984). A comparison of Fourier methods for the description of wing shape in mosquitoes (Ritera culicidae). Syst Zool. 33:302-317.

Sesa-Nogueras, E., Faundez-Zanuy,M (2012) Biometric recognition using online uppercase handwritten text. Pattern Recognition 45, 128-144.

Tracey, S.R., Lyle, J.M., Duhamelb, G. (2006) Application of elliptical Fourier analysis of otolith form as a tool for stock identification. Fisheries Research 77, 138-147

Tort, A. (2003) Elliptical Fourier Functions as a Morphological Descriptor of the Genus Stenosarina (Brachiopoda, Terebratulida, New Caledonia) Math. Geology, 35 (7), 873-885. 